\title{
Numerical study of fluid filtration in three-layer interacting pressure porous formations
}

\author{
Normakhmad Ravshanov ${ }^{1^{*}}$, Zafar Abdullaev ${ }^{2}$, Sabur Aminov ${ }^{3}$ and Otabek Khafizov ${ }^{2}$ \\ ${ }^{1}$ Research Institute for the Development of Digital Technologies and Artificial Intelligence, Tashkent, \\ Uzbekistan \\ ${ }^{2}$ Tashkent Institute of Irrigation and Agricultural Mechanization Engineers, Tashkent Uzbekistan \\ ${ }^{3}$ Tashkent State Agrarian University, Tashkent Uzbekistan
}

\begin{abstract}
A mathematical model was developed to numerically study the process of fluid and gas filtration in three-layer interacting pressure porous formations. It is based on the reviews related to the mathematical modeling developed in recent years; it describes a system of partial differential equations with boundary and initial conditions and a conservative numerical algorithm for conducting a computational experiment (CE) on a computer. In this article, to analyze and make managerial decisions, the results of numerical calculations are presented in the form of graphical objects, which can be used to propose schemes for the location and capacity of vertical drainage wells to protect irrigated and non-irrigated areas from flooding. Using the proposed mathematical tool, it is possible to obtain the predicted groundwater levels of any area for the required period of time, taking into account some factors, such as the inhomogeneity of the reservoir in a plan, the slope of the confining layer, and other hydrogeological, hydro-technical, and natural conditions, or to calculate the thickness and the optimal location of vertical drainage wells to protect the territory, and to develop oil and gas fields and increase their oil and gas recovery. Analysis of the results of a numerical study of the process made it possible to establish the degrees of influence of the elastic filtration regime in a weakly permeable layer on the overflow into adjacent layers.
\end{abstract}

\section{Introduction}

Special attention is paid worldwide to meet the growing demand for petroleum products. The fuel and energy industry plays a vital role in the economic development of any state, and the oil and gas industry has made an unprecedented leap in history.

Models of filtration of inhomogeneous liquid in porous media and methods of their numerical study serve as a theoretical basis for developing and improving the efficiency of oil and gas production, design and control of complex technological processes, and protection of groundwater from pollution, etc. Therefore, the development of adequate mathematical models of filtration of inhomogeneous liquid and transfer of substances in porous media is one of the urgent problems of fluid and gas mechanics.

*Corresponding author: ravshanzade-09@mail.ru 
At present, the basic issues in research of the mechanisms of fluid filtration, the transfer of suspended particles of suspension in porous media, the interaction of these particles with the surface of the skeleton of a porous medium are the construction of mathematical models of nonlinear anomalous processes of fluid and gas filtration, and their numerical study, which is an important aspect in the theory of filtration.

It should be noted that significant results were obtained in the field of mathematical modeling of filtration and transfer of substances, inhomogeneous fluids in porous media.

An analysis of research articles and literary sources devoted to mass transfer problems in gas-dynamic and filtration processes shows that significant results of a theoretical and practical nature have been obtained in the past 10 years.

In particular, in [1], within the framework of the two-fluid approach, a mathematical model was developed for the filtration process of suspended matter in the surrounding area of water flooded wells. The article discusses two mechanisms that affect the transfer of non-colloidal fine particles: capture and mobilization. As the authors note, the captured particles reduce the permeability and porosity of the rock, which leads to a decrease in the injection capacity of the well. The model contains a minimum of setting parameters describing the capture and mobilization rates of particles. Calibration of the model is made based on existing experimental (laboratory) data. A parametric study of various flooding scenarios is conducted using calculating experiments on a computer.

The study in [2] presents a new quick assessment method to determine the susceptibility to suffusion based on the general curvature of particle size distribution. The farther the particle size distribution deviates from its centerline, the more likely it is to behave severely. In this article, the new assessment method was tested on experimental data, and the results showed a good correlation.

In [3], the process of internal erosion in a three-phase saturated soil was studied. The mathematical model describing this process consists of the equations of conservation of mass, Darcy's law, and the capillary pressure equation. The original system of equations is reduced to a system of three equations for porosity, pressure, and water saturation. The equation of water saturation obtained is a degenerate equation. The degenerate problem in the one-dimensional domain is solved numerically by the finite difference method.

In [4], a mathematical model of the multiscale process of liquid filtration in porous media is presented. The mathematical model was developed based on the three-dimensional Navier-Stokes equation with the Carro-Yasuda viscosity using the method of asymptotic averaging. To solve local problems, a numerical algorithm was developed for nonNewtonian fluids in periodic cells, and the distribution of velocity, pressure and viscosity in an individual pore was obtained. An algorithm for calculating the permeability tensor was developed, and the effects of fluid rheology were highlighted.

A mathematical model was developed in [5] for a comprehensive study of the process of gas filtration in a porous medium. The model was described by a nonlinear partial differential equation and the corresponding boundary and internal conditions. The article presents the main stages of developing a mathematical model of the gas filtration process in porous media, taking into account changes in the hydrodynamic parameters of the object. The following numerical methods were used to solve this problem: locally-one-dimensional schemes and schemes of longitudinal-transverse direction. Several methods for constructing an iterative process were tested to solve the problem of nonlinear gas filtration in a porous medium.

In [6], a mathematical model was developed for non-isothermal gas filtration taking into account the phase transition. An algorithm for solving the problem was constructed; it calculates the basic parameters of the thermal impact on a hydrate-saturated reservoir, taking into account the dissociation of gas hydrate. 
In [7], a fractional-differential mathematical model of the process of anomalous geomigration based on the MIM approach (mobile-immobile medium) was used in engineering practice, where the process of modeling the object of research was simplified. When formulating the problem, two-dimensional initial-boundary conditions are set for convective diffusion of soluble substances under conditions of vertical stationary filtration of groundwater with a free surface from the reservoir to the coastal drain. To simplify the modeling domain, the technique of transition to the domain of complex flow potential through conformal mapping was used. In the case of averaging the filtration rate over the region of the complex potential of the flow, an analytical solution to the problem under consideration was obtained. A numerical algorithm for solving the problem was developed for the case of variable filtration rate.

The study in [8] discusses a solution related to the technological process of filtration and dehydration of liquid solutions from small particles and unwanted ionic compounds. A mathematical model and a numerical algorithm were developed, taking into account key factors and parameters that significantly affect the process under consideration as a whole. The article resolves the problems associated with determining the basic parameters and ranges of their change. Based on the analysis of the conducted numerical experiments, conclusions are formulated that serve as the basis for making appropriate managerial decisions.

A mathematical model of gas production from a reservoir, initially saturated with methanol and its hydrate, under conditions of negative (below $0^{\circ} \mathrm{C}$ ) initial reservoir temperature is presented in [9]. An algorithm is proposed, and a numerical scheme is constructed that allows determining the basic parameters of a non-isothermal filtration flow in a hydrate-saturated reservoir, taking into account the decomposition of the hydrate into gas and ice. The analysis of the influence of the mass flow rate of the recoverable gas, the permeability of the porous medium, the initial formation temperature, and the initial hydrate saturation on the mode and rate of gas hydrate decomposition is conducted

The problem of modeling the dynamics of a locally non-equilibrium in time process of convective diffusion of soluble substances under conditions of plane-vertical stationary filtration of groundwater with a free surface, taking into account the presence of interphase mass transfer, is considered in [10]. The relevance of solving these problems is associated, in particular, with the need to develop measures for soil washing, desalination, and purification of groundwater from pollutants. For mathematical modeling of the corresponding transfer process in environments with the property of temporal non-locality, the apparatus of integro-differentiation of fractional order was used.

The study of inverse problems of recovering the coefficients of a linear pseudoparabolic equation arising in filtration theory was considered in [11], where Neumann-type boundary conditions are supplemented by overdetermination conditions, which are the values of the solution at some interior points of the domain. The existence and uniqueness of theorems for solutions in Sobolev spaces are proven in the article.

In [12], computer simulation of filtration processes based on the laws of hydrodynamics is considered. The adequacy of the developed models was verified by conducting a series of computational experiments. Created mathematical and software that serves the purpose of research, forecasting, and decision-making in the development and design of oil and gas fields.

Determination of dynamic indices of production in the oil and gas deposits development is associated with the solution of boundary value problems of unsteady filtration of a threephase mixture during the mixed flow of oil, gas, and water in the reservoir. Since small oil and gas fields in most cases are developed with a few production-and-injection wells randomly located in the reservoir, the filtration process cannot be systematized as a onedimensional flow. 
Consequently, the determination of pressure fields and rock saturation with water, oil, and gas is one of the important but difficult tasks to be solved when drawing up technological schemes for the development of oil and gas deposits.

Depending on the various combinations of fluid phases in a porous medium, different formulations of joint filtration of multiphase fluids can be considered.

Having analyzed the results obtained above, the authors of this research consider the process of fluid filtration in multilayer interacting pressure porous formations when there is an unsteady fluid flow to the circle and an on line (straight) gallery of wells.

\subsection{Problem Statement}

Let us consider the problem of unsteady flow to on line (straight) galleries in a three-layer bounded formation.

Let one battery of perfect vertical drains be drilled into well-permeable layers, each with a radius $R_{c_{1}}, R_{c_{2}}$. We will assume that the three-layer formation is limited in plan and has the shape of a circle with a radius $R_{s}$, and it is impermeable. Suppose that the wells on batteries are located and operated in such a way that the work of the gallery can replace their work.

Then, due to radial symmetry, this problem is reduced to the integration of the system of partial differential equations with corresponding initial, boundary, and internal conditions; that is, the fluid flow in such a three-layer formation, taking into account the elastic regime in a weakly permeable layer and the process of unsteady fluid flow to the central well can be presented as:

$$
\begin{aligned}
& \frac{1}{a_{1}} \frac{\partial H_{1}}{\partial t}=\frac{1}{r} \frac{\partial}{\partial r}\left(r \frac{\partial H_{1}}{\partial r}\right)-\frac{k}{T_{1}} \frac{\partial H\left(r, m+m^{\prime \prime}, t\right)}{\partial z}, \\
& \frac{1}{a} \frac{\partial H}{\partial t}=\frac{\partial^{2} H}{\partial z^{2}}, \\
& \frac{1}{a_{2}} \frac{\partial H_{2}}{\partial t}=\frac{1}{r} \frac{\partial}{\partial r}\left(r \frac{\partial H_{1}}{\partial r}\right)+\frac{k}{T_{2}} \frac{\partial H\left(r, m^{\prime \prime}, t\right)}{\partial z}
\end{aligned}
$$

With the following initial and boundary conditions:

$$
\begin{aligned}
& H_{1}(r, 0)=H_{10}, H(r, \mathrm{z}, 0)=H_{0}, H_{2}(r, 0)=H_{20}, \\
& \frac{\partial H_{1}\left(R_{c_{1}}, t\right)}{\partial r}=\frac{Q_{1}}{2 \pi T_{1} R_{c_{1}}}, \frac{\partial H_{1}\left(R_{s}, t\right)}{\partial r}=0 \\
& H\left(r, m^{\prime \prime}, t\right)=H_{2}(r, t), H\left(r, m+m^{\prime \prime}, t\right)=H_{1}(r, t) \\
& \frac{\partial H_{2}\left(R_{c_{2}}, t\right)}{\partial r}=\frac{Q_{2}}{2 \pi T R_{c_{2}}} \frac{\partial H_{2}\left(R_{s}, t\right)}{\partial r}=0
\end{aligned}
$$


where $H_{10}, H_{0}, H_{20}$ are the given functions, $Q=Q_{1}+Q_{2}$ is the flow rate at the well, $T_{1}, T_{2}$ are the conductivity of the upper and lower well-permeable layers, respectively, $a_{1}$, $a_{2}$ are the piezo- conductivity coefficients of the well-permeable layers, $k$ and $a$ are the filtration coefficient and piezo-conductivity of the weakly permeable layer, $m^{\prime}, m^{\prime \prime}$ are the thicknesses of the upper and lower layers, respectively.

Let in each well-permeable layer $N_{i}=(i=1,2)$ the galleries operate with flow rate $Q_{i l}\left(l=1,2, \ldots, N_{i}\right)$.

If we introduce dimensionless quantities according to the following formulas:

$$
\begin{gathered}
H_{1}^{*}=\frac{H_{1}}{H_{x a p}}, H_{2}^{*}=\frac{H_{2}}{H_{x a p}}, H^{*}=\frac{H}{H_{x a p}} \\
\xi=\frac{x}{L}, \eta=\frac{z}{m}, \quad \xi_{\Gamma l}=\frac{L_{1 \Gamma l}}{L}, \xi_{\Gamma e}^{\prime}=\frac{L_{2 \Gamma l}}{R_{K}}, \tau=\frac{Q_{2}}{R_{K}^{2}} t
\end{gathered}
$$

then, we obtain a mathematical model of the process of fluid filtration in three-layer interacting pressure porous formations:

$$
\begin{aligned}
& \frac{1}{a} \frac{\partial H_{1}}{\partial t}=\frac{\partial^{2} H_{1}}{\partial x^{2}}-\frac{K}{T_{1}} \frac{\partial H\left(x, m+m^{\prime \prime}, t\right)}{\partial z} \\
& \frac{1}{a} \frac{\partial H}{\partial t}=\frac{\partial^{2} H}{\partial z^{2}} \\
& \frac{1}{a_{2}} \frac{\partial H_{2}}{\partial t}=\frac{\partial^{2} H_{2}}{\partial x^{2}}+\frac{K}{T_{2}} \frac{\partial H\left(x, m^{\prime \prime}, t\right)}{\partial z}
\end{aligned}
$$

with the following initial and boundary conditions:

$$
\begin{aligned}
& H_{1}(x, 0)=H(x, z, 0)=H_{2}(x, t)=H_{0} \\
& \frac{\partial H_{i}(0, t)}{\partial x}=0 \frac{\partial H_{i}(L, t)}{\partial x}=0 \\
& \frac{\partial H_{i}\left(L_{\Gamma_{i}}+0, t\right)}{\partial x}-\frac{\partial H_{i}\left(L_{\Gamma_{i}}-0, t\right)}{\partial x}=\frac{Q_{\Gamma_{i}}}{T_{i} l}, \\
& H\left(x, m^{\prime \prime}, t\right)=H_{2}(x, t), \quad H\left(x, m+m^{\prime \prime}, t\right)=H_{1}(x, t),
\end{aligned}
$$

where $i=1,2$.

Then the system of differential equations (10) - (12) with initial and boundary conditions (13) - (18) is rewritten as 


$$
\begin{aligned}
& \alpha \frac{\partial H_{1}^{*}}{\partial \tau}=\frac{\partial^{2} H_{1}^{*}}{\partial \xi^{2}}-\sigma_{1} \frac{\partial H^{*}}{\eta} \\
& \beta \frac{\partial H^{*}}{\partial \tau}=\frac{\partial^{2} H^{*}}{\partial \eta^{2}} \\
& \frac{\partial H_{2}^{*}}{\partial \tau}=\frac{\partial^{2} H_{2}^{*}}{\partial \xi^{2}}+\sigma_{1} \frac{\partial H^{*}}{\partial \eta} \\
& H_{1}^{*}(\xi, 0)=H_{10}^{*} \text {, } \\
& \frac{\partial H_{1}^{*}(0, \tau)}{\partial \xi}=0 \quad \frac{\partial H_{1}^{*}(1, \tau)}{\partial \xi}=0 \\
& \frac{\partial H_{1}^{*}\left(\xi_{\Gamma l}+0, \tau\right)}{\partial \xi}-\frac{\partial H_{1}^{*}\left(\xi_{\Gamma l}-0, \tau\right)}{\partial \xi}=Q_{\Gamma l}^{*} \quad H^{*}(\xi, \eta, 0)=H_{0}^{*} \\
& H^{*}\left(\xi, \frac{m^{\prime \prime}}{m}, \tau\right)=H_{2}^{*}(\xi, \tau) \quad H^{*}\left(\xi, \frac{m+m^{\prime \prime}}{m}, \tau\right)=H_{2}^{*}(\xi, \tau) \\
& H_{2}(\xi, 0)=H_{20}^{*} \frac{\partial H_{2}^{*}(0, \tau)}{\partial \xi}=0 \frac{\partial H_{2}^{*}(1, \tau)}{\partial \xi}=0 \text {, } \\
& \frac{\partial H_{2}^{*}\left(\xi_{\Gamma l}^{\prime}+0, \tau\right)}{\partial \xi}-\frac{\partial H_{2}^{*}\left(\xi_{\Gamma l}^{\prime}-0, \tau\right)}{\partial \xi}=Q_{2 l}^{*} \quad l=1,2, \ldots, N_{2}
\end{aligned}
$$

Let us introduce the functions

$$
H_{1}^{*}=U-\varphi_{1}(\xi), H_{2}^{*}=V-\varphi_{2}(\xi)
$$

where

$$
\varphi_{1}(\xi)=\frac{1}{2} \xi^{2} \sum_{l=1}^{N_{1}} Q_{1 l}^{*}-\sum_{l=1}^{N_{1}} \alpha_{1 l} Q_{1 l}^{*}\left(\xi-\xi_{\Gamma l}\right) \quad \varphi_{2}(\xi)=\frac{1}{2} \xi^{2} \sum_{l=1}^{N_{2}} Q_{2 l}^{*}-\sum_{l=1}^{N_{2}} \beta_{l} Q_{2 l}^{*}\left(\xi-\xi_{\Gamma l}^{\prime}\right) .
$$

If we take into account (24), then the system of equations (15) - (17) with conditions (18) - (23) is rewritten as

$$
\begin{aligned}
& \alpha \frac{\partial U}{\partial \tau}=\frac{\partial^{2} U}{\partial \xi^{2}}-\sigma_{1}\left[\left(U-\varphi_{1}\right)-\left(V-\varphi_{2}\right)+\delta \frac{\partial W\left(\xi, \frac{m+m^{\prime \prime}}{m}, \tau\right)}{\partial \xi}\right] \\
& \frac{\partial W}{\partial \tau}=\frac{1}{\beta} \frac{\partial^{2} W}{\partial \xi^{2}}-\left(\eta-\frac{m^{\prime \prime}}{m}\right) \frac{\partial U}{\partial \tau}-\left(1-\eta+\frac{m^{\prime \prime}}{m}\right) \frac{\partial V}{\partial \tau}
\end{aligned}
$$




$$
\begin{aligned}
& \frac{\partial V}{\partial \tau}=\frac{\partial^{2} V}{\partial \xi^{2}}+\sigma_{2}\left[\left(U-\varphi_{1}\right)-\left(V-\varphi_{2}\right)+\delta \frac{\partial W\left(\xi, \frac{m^{\prime \prime}}{m}, \tau\right)}{\partial \eta}\right] \\
& U(\xi, 0)=H_{10}^{*}+\varphi_{1}(\xi) \\
& \frac{\partial U(0, \tau)}{\partial \xi}=0 \frac{\partial U(1, \tau)}{\partial \xi}=0 \\
& W\left(\xi, \frac{m^{\prime \prime}}{m}, \tau\right)=0 \quad W\left(\xi, \frac{m+m^{\prime \prime}}{m}, \tau\right)=0 \\
& W(\xi, \eta, 0)=H_{0}^{*}-\left(\eta-\frac{m^{\prime \prime}}{m}\right) H_{10}^{*}-\left(1-\eta+\frac{m^{\prime \prime}}{m}\right) H_{20}^{*} \\
& V(\xi, 0)=H_{2}^{*}+\varphi_{2}(\xi) \frac{\partial V(0, \tau)}{\partial \xi}=0 \frac{\partial V(1, \tau)}{\partial \xi}=0
\end{aligned}
$$

\section{Methods}

From system (25) - (27) and conditions (28) - (32), we see that it is possible to apply the method of numerical solution of the problem described in [13],[14], and the algorithm is suitable here if $Q_{i}^{*}=0,(i=1,2)$ and the region of continuous variation of the argument is $\eta_{c} \leq \eta \leq 0,0 \leq \tau \leq T<\infty$ is replaced with a finite set of points (grid) with coordinates, $\left\{\eta_{i}=\eta_{c}+i \Delta \eta, \tau_{j}=j \Delta \tau, i=0,1,2, \ldots, I ; j=1,2, \ldots\right\}$, where $\Delta \eta, \Delta \tau$ are the grid steps.

The derivatives entering equations (25) - (32) are replaced with difference relations, and then we obtain the finite-difference equation[15].

$$
\begin{aligned}
& a_{i} u_{i-1}-b_{i} u_{i}+c_{i} u_{i+1}+d_{i} v_{i}=-f_{i}, \\
& a_{i}^{\prime} v_{i-1}-b_{i}^{\prime} v_{i}+c_{i}^{\prime} v_{i+1}+d_{i}^{\prime} u_{i}=-f_{i}^{\prime} i=1,2, \ldots, I-1
\end{aligned}
$$

The coefficients of the system equation (33), (34) are given in detail in [33]. Further, solving the system of algebraic equations (33) - (34) by the sweep method[16], we calculate the dynamics of the change in the sought for time variables.

\section{Results and Discussion}

Based on the above-mentioned software and a solution algorithm, a program was developed to conduct a comprehensive study of the process of unsteady inflow to straight and circular galleries. The results of the performed numerical calculations are shown in Figs. 1,2 and in [14]. 
To illustrate the above algorithm, we consider examples with the following reservoir parameters: $a_{1}=a_{2}=1000000 m^{2} /$ day $\quad a=100 m^{2} /$ day $\quad m^{\prime}=m^{\prime \prime}=100$ meter , $m=10$ meter, $K=0,01 m /$ day, $T_{1}=T_{2}=1000 m^{2} /$ day .

The results of the computational experiments (CE) conducted are given at a decrease in pressure

$$
S^{*}=\frac{T\left(H-H_{0}\right) R_{\Gamma}}{Q R_{K}}
$$

and at $\frac{R_{\Gamma}}{R_{K}}=0.3 ; 0.4 ; 0.5 ; 0.6 ; 0.7$; taking into account the elastic regime and the pressure lowering without considering the elastic regime, at $\frac{R_{\Gamma}}{R_{K}}=0.5$.

As follows from the computational experiments (Fig. 1) performed, the pressure in the upper aquifer along the length of the reservoir, considering the elastic regime, increases with time. This growth is especially noticeable when the layer length changes in the range of 0.2-0.4. A similar pattern can be observed in the lower aquifer at $R_{\Gamma} / R_{K}=0.3$.

The analysis of the performed numerical calculations showed that the change in the pressure in the upper and lower layers substantially depends on the value of $R_{\Gamma} / R_{K}$, and with an increase in the value of $R_{\Gamma} / R_{K}$, the pressure in the upper and lower aquifers decreases proportionally.

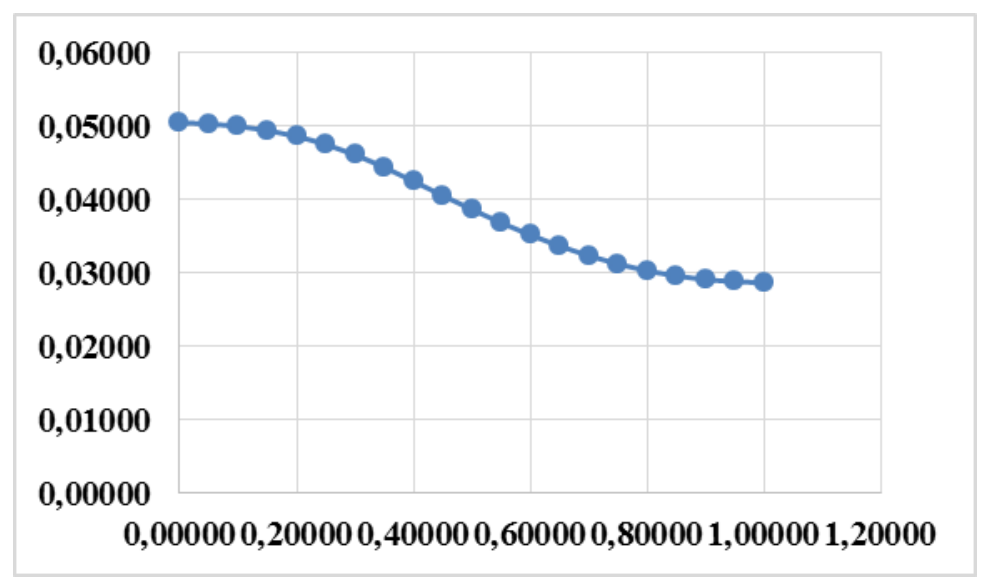

Fig. 1. Change in pressure in the upper aquifer along the length of the reservoir considering the elastic regime (at $R_{\Gamma} / R_{K}=0.3 ; \mathrm{t}=2.0$ day. ) 


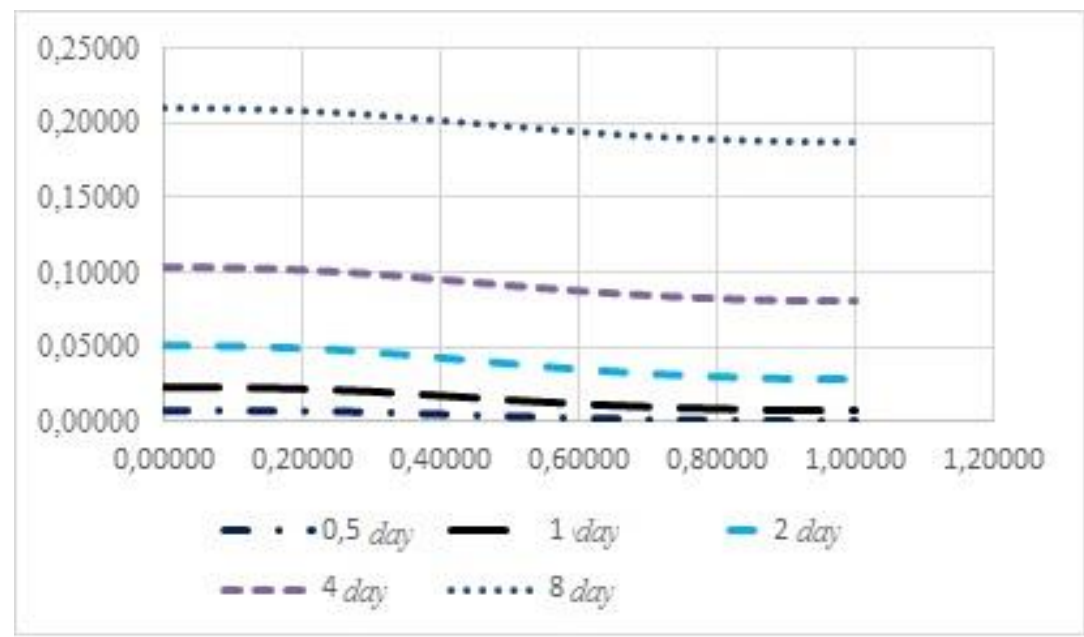

Fig 2. Change in pressure in the lower aquifer along the length of the reservoir considering the elastic regime

With CE, it was determined that under the elastic filtration mode, the pressure of groundwater in the layers grows in an exponential law; this growth is especially noticeable in the lower pressure layer. From numerical calculations on a computer, it was established that for various values of the hydrodynamic parameters of the process, the overflow through the boundary surface of filtration layers of fluids significantly depends on the conductivity of the upper and lower well-permeable layers, the piezoconductivity coefficients of well-permeable layers, and on the filtration coefficients and the piezo conductivity of weakly permeable layer.

Analysis of the results showed that in the upper layer with time, the pressure increases in a stepwise law, and in the lower layer, on the contrary, it decreases similarly with time in a stepwise law.

\section{Conclusion}

To carry out a comprehensive study of the process of fluid filtration in multilayer interacting pressure layers, a mathematical model and a numerical algorithm were developed; computational experiments on a computer were conducted.

The results of numerical calculations of the problems made it possible to establish the degrees of influence of the elastic filtration regime in a weakly permeable layer without overflow into adjacent layers.

Based on the mathematical apparatus developed, it is possible to propose schemes for the location and capacity of vertical drainage wells to protect irrigated and non-irrigated areas from flooding.

Using the proposed mathematical tool, it is possible to obtain the predicted groundwater levels of any area for the required period of time, taking into account some factors, such as the inhomogeneity of the reservoir in a plan, the slope of the confining layer, infiltration recharge or evaporation and other hydrogeological, hydro-technical and natural conditions, and to calculate the capacity and the optimal location of vertical drainage wells to protect the territory, and to develop oil and gas fields. 


\section{References}

1. Tolmacheva K I, Boronin S A and Osiptsov A A, Formation damage and cleanup in the vicinity of flooding wells, Multi-fluid suspension flow model and calibration on lab data J. Pet. Sci. Eng. pp 408-418 (2019).

2. To P, Scheuermann A and Williams D J, Quick assessment on susceptibility to suffusion of continuously graded soils by curvature of particle size distribution Acta Geotech. 5 1241-8. (2018).

3. Sibin A, Numerical study of a mathematical model of internal erosion of soil Journal of Physics, Conference Series. (2017).

4. Dimitrienko Y.I and Li.S, Mathematical Simulation of local transfer for nonNewtonian uid in porous fabrics J. Phys. Conf. Ser. 1392 (1-6). (2019).

5. Ravshanov N, Aminov S and Kravets O J, Mathematical model and numerical algorithms to analyze gas filtration process in a porous medium Journal of Physics, Conference Series. (2019).

6. Musakaev N G, Borodin S L and Belskikh D S, Mathematical modeling of thermal impact on hydrate-saturated reservoir J. Comput. Methods Sci. Eng. 20 pp 43-51. (2020).

7. Bohaienko V and Bulavatsky V, Simplified mathematical model for the description of anomalous migration of soluble substances in vertical filtration flow Fractal Fract. 4 pp 1-11. (2020).

8. Ravshanov N, Saidov U, Karshiev D and Bolnokin V. E, Mathematical model and numerical algorithm for studying suspension filtration in a porous medium considering the processes of colmatation and suffusion IOP Conference Series, Materials Science and Engineering. (2020)

9. Musakaev N. G, Khasanov M. K and Borodin S. L, The mathematical model of the gas hydrate deposit development in permafrost Int. J. Heat Mass Transf. 118, pp 45561. (2018).

10. Bohaienko V. A and Bulavatsky V. M, Computer simulation based on non-local model of the dynamics of convective diffusion of soluble substances in the underground filtration flow under mass exchange conditions J. Autom. Inf. Sci. 51 pp 16-29. (2019).

11. Shergin S.N, Safonov E.I and Pyatkov S.G, On some inverse coefficient problems with the pointwise overdetermination for mathematical models of filtration Bull. South Ural State Univ. Ser. Math. Model. Program. Comput. Softw. 12 pp 82-95. (2019).

12. Kurbonov $\mathrm{N}$ and Aminov S. Computer modeling of filtration processes with piston extrusion Journal of Physics, Conference Series (2020).

13. Ravshanov N, Nazirova E.S and Pitolin V. M, Numerical modelling of the liquid filtering process in a porous environment including the mobile boundary of the "oilwater" section Journal of Physics, Conference Series, (2019).

14. Ravshanov N., Nazirova E.Sh., Oripzhanova U. A S . Problems of computational and applied mathematics Probl. Comput. Appl. Math. 25 pp 28-49. (2020).

15. Ravshanov N, Khurramov I and Aminov S M, Mathematical modeling of the process of water-soline transport in soils J. Phys. Conf. Ser. 1210 (2019).

16. Aminov S, Rajabov N, Azamov $\mathrm{T}$ and Ravshanov Z, Numerical study of salt-transfer process in soils Int. J. Adv. Trends Comput. Sci. Eng. (2020). 